\title{
Correction to: A multiparametric analysis based on DCE-MRI to improve the accuracy of parotid tumor discrimination
}

\author{
Zhifeng Xu ${ }^{1}(\mathbb{D}) \cdot$ Shaoyan Zheng ${ }^{2} \cdot$ Aizhen Pan $^{1} \cdot$ Xiaofang Cheng $^{3} \cdot$ Mingyong Gao $^{1}$ \\ Published online: 4 February 2020 \\ (C) Springer-Verlag GmbH Germany, part of Springer Nature 2020
}

Correction to: European Journal of Nuclear Medicine and Molecular Imaging (2019) 46:2228-2234. https://doi.org/10.1007/s00259-019-04447-9

Figure captions of Figures 2, 3, and 4 were incorrect in the original version of this article. Correct captions are presented here:

Fig. 2 PA in the left parotid. a Axial T1WI showing isointense nodules. b Axial T2WI showing mixed hyperintense and isointense nodules. c DCE-T1WI showing nonhomogeneous enhanced nodules. d Type A TIC

Fig. 3 Warthin's tumor in the right parotid gland. a Axial T1WI tumor showing an isointense signals. b Axial T2WI the nodule showing slightly hyperintense and isointense signals. $\mathbf{c}$ Nodule showing slight homogeneous enhancement on DCE T1WI. d Type B TIC

Fig. 4 Lymphoepithelial carcinoma in the left parotid gland. a Axials T1WI showing isointense nodules. b Axial T2WI of nodule showing slight hyperintense and isointense areas. c DCE-T1WI showing a slight homogeneous enhancement. d Type C TIC

This article is part of the Topical Collection on Erratum.

The online version of the original article can be found at https://doi.org/ 10.1007/s00259-019-04447-9

Zhifeng Xu

xuzf83@126.com

1 Department of Radiology, The First People's Hospital of Foshan, Foshan, Guangdong, China

2 Department of MR, Shantou Central Hospital, Shantou, Guangdong, China

3 Department of Radiology, Affiliated Brain Hospital of Guangzhou Medical University (Guangzhou Huiai Hospital),

Guangzhou, People's Republic of China 